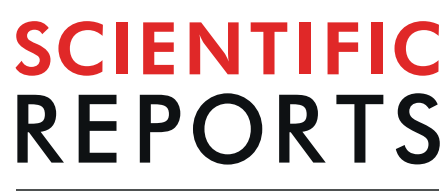

natureresearch

Received: 5 May 2019

Accepted: 17 September 2019

Published online: 03 October 2019

\section{Whale counting in satellite and aerial images with deep learning}

\author{
Emilio Guirado $\mathbb{B}^{1,2}$, Siham Tabik ${ }^{1}$, Marga L. Rivas ${ }^{3}$, Domingo Alcaraz-Segura $\mathbb{B}^{2,4}$ \& \\ Francisco Herrera ${ }^{1}$
}

Despite their interest and threat status, the number of whales in world's oceans remains highly uncertain. Whales detection is normally carried out from costly sighting surveys, acoustic surveys or through high-resolution images. Since deep convolutional neural networks (CNNs) are achieving great performance in several computer vision tasks, here we propose a robust and generalizable CNN-based system for automatically detecting and counting whales in satellite and aerial images based on open data and tools. In particular, we designed a two-step whale counting approach, where the first CNN finds the input images with whale presence, and the second CNN locates and counts each whale in those images. A test of the system on Google Earth images in ten global whale-watching hotspots achieved a performance (F1-measure) of $81 \%$ in detecting and $94 \%$ in counting whales. Combining these two steps increased accuracy by $36 \%$ compared to a baseline detection model alone. Applying this cost-effective method worldwide could contribute to the assessment of whale populations to guide conservation actions. Free and global access to high-resolution imagery for conservation purposes would boost this process.

Whales, which comprise some of the largest animals that have ever existed, have always thrilled humans ${ }^{1,2}$. Whales had and keep an enormous economic and societal value ${ }^{3}$. More than 13 million whale-watchers were registered in 2008 across 119 countries, generating a global economic activity of US\$ 2.1 billion $^{4}$. Since whales generally are long-living species at high trophic-levels, they play an essential role to structure marine food webs, and to maintain ecosystem functions and services ${ }^{5,6}$. In the past, commercial whaling depleted whale populations from $66 \%$ to $90 \%$ from their original numbers, which subsequently caused alterations in marine biodiversity and functions ${ }^{7}$. To prevent whales from extinction, the signatories of the International Convention for the Regulation of Whaling limited whale hunting to scientific or aboriginal actions since 1946, although the moratorium on commercial whaling did not come into force until $1982^{8}$. Even though, there still exist a great uncertainty around the number of whales in the oceans and the viability of their populations ${ }^{9}$. In late 2017, the Species Red List of the International Union for Conservation of Nature (IUCN) $)^{10}$ reported that $22 \%$ of 89 evaluated cetacean species were classified as threatened, whereas almost $50 \%$ species could not be evaluated due to the lack of data. Hence, a more accurate estimation of whale distribution and population sizes is essential to warrant cetacean conservation ${ }^{11}$.

The process of identifying and estimating the number of cetaceans is normally carried out $\mathrm{t}^{12,13}$ (1) in situ, from ships $^{14,15}$, planes ${ }^{16,17}$ or ground stations ${ }^{18}$, by using visual surveys ${ }^{19,20}$, acoustic methods ${ }^{21,22}$, or a combination of both ${ }^{23,24}$; or (2) ex situ, by using satellite tracking ${ }^{25,26}$ or photo-interpretation or classical image classification techniques on Very High Resolution (VHR) aerial or satellite images ${ }^{27-31}$. However, these methods are costly, not robust against scenario changes (e.g., different regions or atmospheric conditions), not generalizable to a massive set of images, and often require handcrafted features ${ }^{32,33}$. Indeed, biodiversity conservation would certainly benefit from robust and automatic systems to assess species distributions, abundances and movements from satellite and aerial images ${ }^{34,35}$.

Deep learning methods, particularly Convolutional Neural Networks (CNNs), could help in this sense since $\mathrm{CNNs}$ are already outperforming humans in visual tasks such as image classification and object detection ${ }^{36}$. CNN models have the capacity to automatically learn the distinctive features of different object classes from a large

\footnotetext{
${ }^{1}$ Andalusian Research Institute in Data Science and Computational Intelligence, University of Granada, 18071, Granada, Spain. ${ }^{2}$ Andalusian Center for the Assessment and Monitoring of Global Change, University of Almería, 04120, Almería, Spain. ${ }^{3}$ Department of Biology, University Marta Abreu of Las Villas, 50100, Santa Clara, Cuba. ${ }^{4}$ iecolab. Interuniversity Institute for Earth System Research, University of Granada, 18071, Granada, Spain. Correspondence and requests for materials should be addressed to E.G. (email: e.guirado@ual.es) or S.T. (email: siham@ugr.es)
} 
number of annotated images to later make correct predictions on new images ${ }^{37}$. Although the construction of a dataset for training is costly, the learning of CNNs on small datasets can be boosted by data-augmentation, which consists of increasing the volume of the training dataset artificially, and additionally by transfer learning, which consists of starting the learning of the network from a prior knowledge rather from $\mathrm{scratch}^{38,39}$.

Identifying whales from aerial and satellite images using CNNs at a global scale is very challenging for several reasons: (1) comprehensive datasets with VHR images of whales to train CNNs do not exist yet; (2) VHR images are expensive and relatively scarce in the marine environment; (3) whales could potentially be confused with other objects such as boats, rocks, waves, or foam; (4) whale postures or behaviour captured in a snapshot are quite variable since different parts of whale bodies can be emerged or submerged (e.g. blowing, logging, etc.); and (5) occlusions and noise could occur due to clouds, aerosols, haze, sunglint, or water turbidity.

In this work, we propose a large scale generalizable deep learning system for automatically counting whales from satellite and aerial images. For this, we combined two CNNs into a two-step model, where the first CNN detects the presence of whales and the second CNN counts the number of whales in the images (See Methods section). To overcome the above mentioned challenges, (1) we combined several open datasets to build an annotated training database of high quality vertical images of whales and of objects that could be confused with whales, (2) we used data augmentation and transfer learning techniques to make the CNNs robust to image variability, (3) we assessed the effect of whale posture and location on the model performance, and, as a proof of concept, (4) we applied the model to free Google Earth coastal imagery in 10 whale-watching hotspots. Additionally, we compared the performance of our combined approach to the performance reached just using the second CNN alone.

\section{Contributions}

The main contributions of this work can be summarized as follows:

- It presents the first proof of concept on how deep learning can be exploited for counting whales in RGB aerial and satellite images and using free machine learning software.

- It addresses the problem of whale counting at large scale areas by using a two-step approach: (1) the first step $\mathrm{CNN}$ selects the candidate images with a high probability of whale presence, and (2) the second step CNN analyzes these images by a detection model to localize and count the existing whales. Combining these two steps increased accuracy by $36 \%$ compared to the baseline detection model alone.

- It provides two datasets that guarantee a good learning for the first-step and second-step CNN-based models, with 2,100 images. For the external evaluation, this work also provides a new test dataset made of 13,348 images of ten marine mammal hotspots.

- It analyzes the effect of whale postures or behavior on model performance.

- It provides evidence on how a CNN based system trained on higher resolution aerial images of whales is able to find whales in lower resolution satellite images.

Preliminaries on CNN models for image classification and object detection in images. Deep Neural Networks (DNNs) are a subset of machine learning algorithms able to learn from a training dataset to make predictions on new examples called testset. They are built using a hierarchical architecture of increasing sophistication, each level of this hierarchy is called layer. One of their main particularities is their capacity to extract the existent features from data automatically without the need of external hand crafted features. Under the supervised learning paradigm, DNNs provide a powerful framework when trained on a large number of labelled samples.

Convolutional Neural Networks (CNNs) are a specialized type of neural networks capable of extracting spatial patterns from images. Their architecture is built by staking three main types of layers: (1) convolutional layer, which is used to extract features at different levels of the hierarchy, (2) pooling layer, which is essentially a reduction operation used to increase the abstraction level of the extracted features and (3) fully connected layer, which is used as a classifier at the end of the pipeline.

CNNs need a large number of examples to achieve good learning. However, building dataset from scratch is costly and time consuming. To overcome these limitations in practice, two techniques are used: Transfer learning and data-augmentation. Transfer-learning consists of using the knowledge acquired in problem A to problem B. This is implemented by initializing the weights of the model for problem B using the pre-trained weights on problem A. Data-augmentation consists of applying specific transformations to the training images. In general, these transformations simulate the deformations that data could suffer in real world, e.g., scaling, rotations, translations, different illumination conditions, cropping parts of the image. It was demonstrated in several works that data-augmentation increases the robustness and generalization capacity of $\mathrm{CNNs}^{40}$.

CNNs constitute the state-of-the art in all the fundamental tasks in computer vision, e.g., in image classification and object detection in images. In image classification, the $\mathrm{CNN}$ model has to analyze the input image and produce a label that describes its visual content, together with a probability that expresses the confidence of the model. In object detection, the CNN detection model has not only to produce the correct label but also determine by means of a bounding box the region in the input image where the target object is located. Examples of the most accurate and robust models for image classification are Inception ${ }^{41}$ and Inception ResNet ${ }^{42}$. The most accurate detection frameworks are end-to-end object detection models that combine a sophisticated detection technique with one of the most powerful CNN classification models. At present, there exist several detection frameworks that provide good trade-off between accuracy, robustness and speed, such as, Faster-RCNN ${ }^{36}$, YOLO9000 ${ }^{43}$, $\mathrm{FPN}^{44}$, RefineDet ${ }^{45}, \mathrm{DSSD}^{46}$ and Focal Dense Object Detection ${ }^{47}$. Furthermore, several studies are focusing on improving these frameworks on specific remote sensing data ${ }^{48-51}$. In this work, we used Faster-RCNN ${ }^{36}$ based on Inception RenNet v2, as it is the most accurate detection framework according to the this study ${ }^{48}$. 


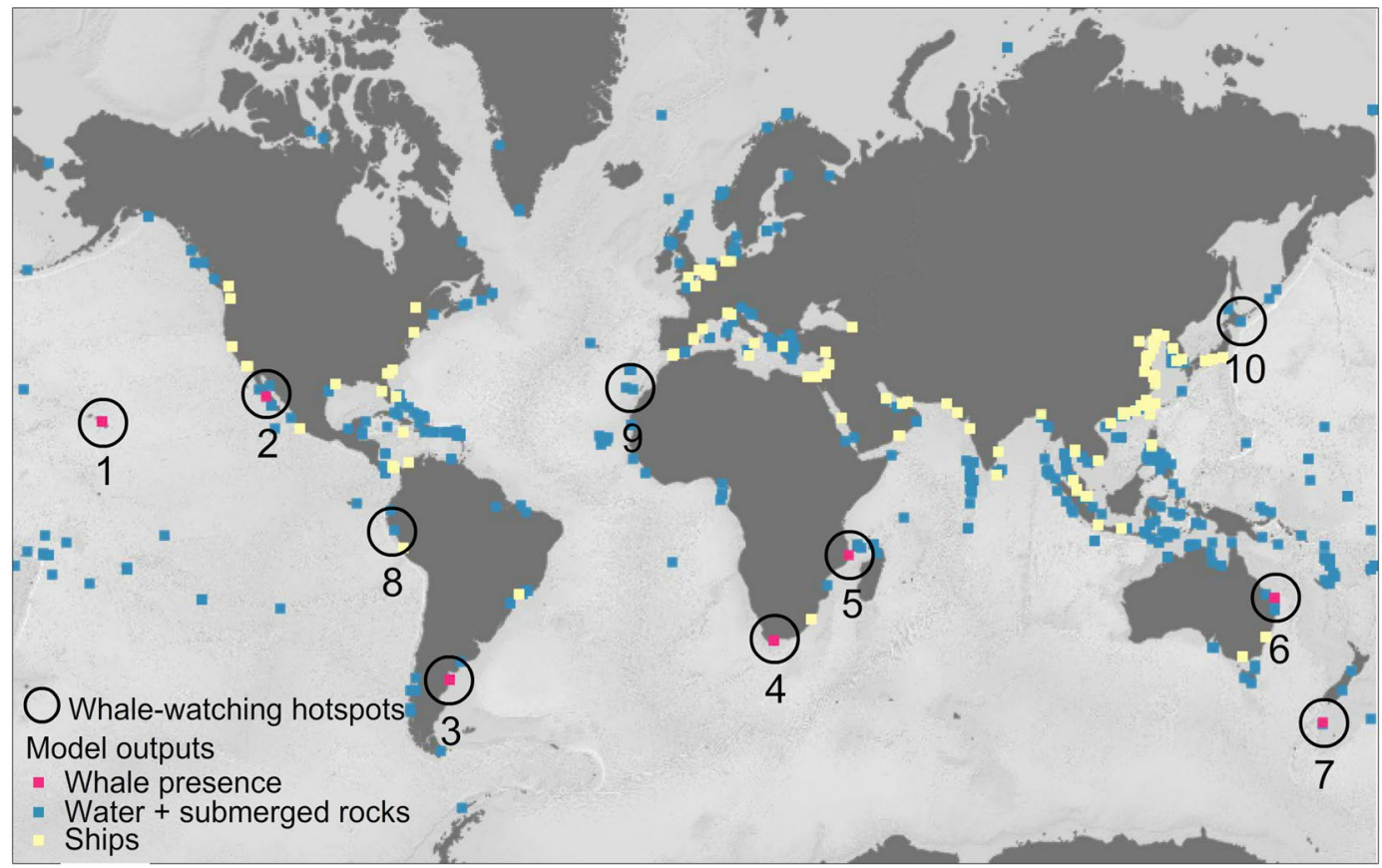

Figure 1. Results at a global scale of the first step whale presence detection model in ten marine mammal hotspots for whale watching (details in Table 1). Red, blue, and yellow cells indicate respectively whale presence, water + submerged rocks, and ships.

\section{Results}

Whale presence detection model (step-1) validation. The analysis of the first step CNN-based model on ten marine mammal hotspots for whale watching (Fig. 1) confirmed the presence of whales in six of the ten assessed whale watching hotspots (Fig. 2). The acquisition dates of the satellite images available through Google Earth for these six sites matched the known whale watching period from the literature (Tables 1,2 ). In the whale watching hotspot located in Memba (Mozambique), the spatial resolution of Google Earth images was not sufficient for the human annotator to determine with a high confidence whale presence and hence, the prediction of the whale presence model was tagged as uncertain. In the three sites where the model did not find whales (Peru, Canary Islands, and Japan), the acquisition date of their Google Earth images was not within the known whale watching period but during the migration season. In the Peruvian coast and in the Canary Islands the detection was particularly challenging since the images presented rough sea.

Step-1 CNN-based model that detects the presence of whales reaches an average F1-measure of $81.8 \%$ for whales, $95.9 \%$ for water + submerged rocks and $96.7 \%$ for ships (Table S2). Only $20.58 \%$ of test grid cells containing whales were misclassified as water $(19.11 \%)$ or ships $(1.47 \%)$. A very small number of water + submerged rocks and ship images were misclassified as whales (1.00\% and $2.25 \%$, respectively; see Fig. 3$)$. An example of a false positive that shows a hand-glider over the sea in Witsand (South Africa) is illustrated in (Fig. S2).

Whales behaviour affects the performance of the first step CNN-based model for detecting the presence of whales (Fig. 3). Higher detectability (greater than $90 \%$ of true positives) was obtained for the following whale postures: blowing, breaching, peduncle, and logging. The lowest detectability occurred for submerged and spyhopping postures (33\% and $60 \%$ of false negative, respectively; see Fig. $4 \mathrm{~A}$ ). Indeed, the lower performance of step-1 model in the Argentinean and New Zealand sites (Table S1, Fig. S1) was due to the much greater frequency of these latter postures in the images (see Data S5). Overall, greater number of whales were in the passive behaviour of logging and submerged (60\% of detected whales and $74 \%$ of photo-interpreted whales), while the lower number of whales were detected under active movements (Fig. 4A, Data S5).

Whale counting (step-2) model validation. The second step CNN-based model for localizing and counting whales analyzes only the cells where step- 1 found whale presence (Fig. 5). From a total number of 84 whales photo-interpreted in this study across six hotspots for whale watching around the world, step-2 automatically localized and counted 62 of them, which gives the model an overall performance of $94 \% \pm 0.015 \%$ of F1 measure (Table S1 and Fig. S3).

\section{Discussion and Conclusions}

This study illustrates how global cetacean conservation could benefit from the operational application of deep learning on VHR satellite images. Using a two-step convolutional neural network model trained with a reduced dataset and applied on free Google Earth imagery, we managed to automatically detect and count 62 whales in six hotspots for whale watching around the world, reaching an overall global F1-measure of $78 \% \pm 0.07 \%$ (F1 measure of $81 \% \pm 0.13 \%$ for presence detection and $94 \% \pm 0.01 \%$ for locating and counting). Our results show how 

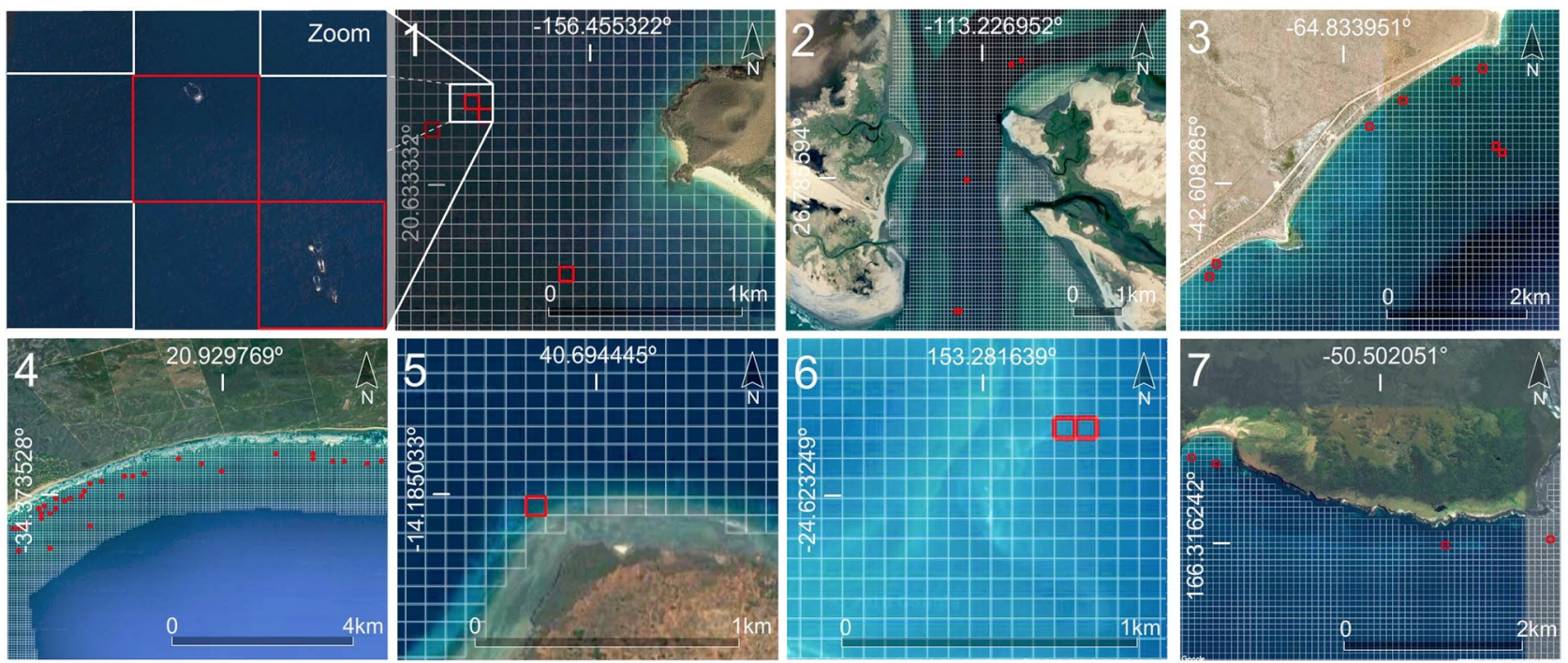

Figure 2. Illustration of the assessed grid cells where the first step CNN-based model detects presence of whales. The cells with whale presence are indicated in red boxes in six of the ten candidate hotspots. In the three remaining hotspots, high resolution images were not available for the whale watching months. Map data: Google, DigitalGlobe.

\begin{tabular}{|l|l|l|l|}
\hline Site IDs Site names (country) & $\begin{array}{l}\text { Cells with whales } \\
\text { (photo-interpreted) }\end{array}$ & $\begin{array}{l}\text { Cells with whales } \\
\text { (CNN-based model) }\end{array}$ & $\begin{array}{l}\text { Total number } \\
\text { of cells }\end{array}$ \\
\hline 1. Hawaiian Islands (USA) & 4 & 4 & 565 \\
\hline 2. Baja California (Mexico) & 7 & 6 & 2,974 \\
\hline 3. Valdés Peninsula (Argentina) & 7 & 4 & 1,295 \\
\hline 4. Witsand (South Africa) & 40 & 35 & 3,312 \\
\hline 5. Memba (Mozambique) & $\mathrm{U}$ & 1 & 594 \\
\hline 6. Coral Sea (Australia) & 2 & 2 & 346 \\
\hline 7. Enderby Island (New Zealand) & 8 & 4 & 790 \\
\hline 8. Peruvian coast (Peru) & 0 & 0 & 1,307 \\
\hline 9. Canary Islands (Spain) & 0 & 0 & 1,045 \\
\hline 10. Japanese coast (Japan) & 0 & 0 & 1,120 \\
\hline Total & 68 & 56 & 13,348 \\
\hline
\end{tabular}

Table 1. Summary of the results of step- $1 \mathrm{CNN}$-model in a total number of 13,348 evaluated cells. The first column shows the IDs and names of the ten evaluated whale watching sites. The second column shows the number of cells with whale presence photo-interpreted by the authors. The third and fourth columns show respectively the number of cells with and without whales according to step-1 CNN-based model predictions in each site. The asterisk and $U$ indicates labeling uncertainty due to the low resolution of the images in that region.

the acquisition date of the satellite image, the behaviour recorded in the image and the resolution of the image can influence whale presence detection and counting. For instance, the spatial resolution of SPOT-6 satellite images was not good enough to assess whether the model was correct in Memba (Mozambique) at the date and location chosen. This robust, transparent and automatic method can have direct and wide implications for whale conservation by assessing whale distributions, abundances, and movement behaviours globally from satellite and aerial images.

Our satellite and aerial based assessment can complement and be compared with other aerial, marine, and land observations. The coastal images of Google Earth at zoom 18 that we used correspond to a visual altitude of $\sim 254 \mathrm{~m}$, similar to the aerial surveys for grey whales, and up to $\sim 4 \mathrm{~km}$ offshore the coast, the maximum distance for whale visual surveys from land $\mathrm{d}^{52}$. In whale assessments, such distances are good enough to get reliable estimates of instantaneous presence and relative population abundances ${ }^{53}$. As new RGB images become available, our method also enables dynamic updates at low cost, to assess seasonal and interannual changes in population sizes, feeding and breeding areas, migratory routes, and distribution ranges around the world.

Several studies show that the performance of CNNs can be equal or even better than humans when the quality of the images is good, for instance, for skin cancer detection ${ }^{54}$, mastering the game of $\mathrm{Go}^{55}$, or generalizing past experiences to new situations ${ }^{56}$. In general, the quality of the images determines the accuracy of the classification in $\mathrm{CNNs}^{57}$, learning and performing better on higher resolution images ${ }^{58}$. However, our results show how $\mathrm{CNN}$-based methods trained on high-quality images (see methods section) can also reach good performance 


\begin{tabular}{|c|c|c|c|c|c|}
\hline $\begin{array}{l}\text { Site IDs Site names } \\
\text { (country) }\end{array}$ & $\begin{array}{l}\text { Latitude Longitude } \\
\text { WGS84 }\end{array}$ & $\begin{array}{l}\text { Whale watching } \\
\text { period }\end{array}$ & $\begin{array}{l}\text { Date of Google } \\
\text { Earth image }\end{array}$ & $\begin{array}{l}\text { Season of } \\
\text { acquisition date }\end{array}$ & $\begin{array}{l}\text { Image source } \\
\text { Pixel size (m) }\end{array}$ \\
\hline 1. Hawaiian Islands (USA) & $20.636602,-156.462511$ & Dec-Apr ${ }^{89}$ & $\begin{array}{l}\text { Apr 3, } 2013 \\
\text { Jan 13, } 2013\end{array}$ & Breeding & $\begin{array}{l}\text { USGS aerial } \\
0.15 \mathrm{M}\end{array}$ \\
\hline $\begin{array}{l}\text { 2. Baja California } \\
\text { (Mexico) }\end{array}$ & $26.769961,-113.242382$ & $\mathrm{Feb}^{90}$ & Feb 20, 2017 & Breeding & $\begin{array}{l}\text { WV-03 } \\
0.31 \mathrm{P} \\
1.24 \mathrm{M}\end{array}$ \\
\hline 3. Valdés Peninsula (Argentina) & $-42.603384,-64.810850$ & May-Dec ${ }^{1}$ & Sep 18, 2003 & Breeding & $\begin{array}{l}\text { QB-02 } \\
0.61 \mathrm{P} \\
2.5 \mathrm{M}\end{array}$ \\
\hline $\begin{array}{l}\text { 4. Witsand } \\
\text { (South Africa) }\end{array}$ & $-34.390203,20.879985$ & $\mathrm{Jul}-O c t^{4}$ & Aug 9, 2009 & Breeding & $\begin{array}{l}\text { GE-01 } \\
0.46 \mathrm{P} \\
1.84 \mathrm{M}\end{array}$ \\
\hline 5. Memba (Mozambique) & $-14.185282,40.691405$ & Jun-Jul ${ }^{91}$ & Jun 23, 2017 & Breeding & $\begin{array}{l}\text { SPOT-6 } \\
1.5 \mathrm{P} \\
6.0 \mathrm{M}\end{array}$ \\
\hline 6. Coral Sea (Australia) & $-24.622170,153.291559$ & Sep-Nov ${ }^{92}$ & Sep 13, 2005 & Breeding & $\begin{array}{l}\text { QB-02 } \\
0.61 \mathrm{P} \\
2.5 \mathrm{M}\end{array}$ \\
\hline 7. Enderby Island (New Zealand) & $-50.501698,166.282294$ & Jul-Sep ${ }^{93}$ & Sep 2, 2012 & Breeding & $\begin{array}{l}\text { WV-02 } \\
0.46 \mathrm{P} \\
1.84 \mathrm{M} \\
\end{array}$ \\
\hline 8. Peruvian coast (Peru) & $-14.253483,-76.159243$ & Jun-Sep ${ }^{94}$ & Mar 9, 2016 & Migrating/Feeding & $\begin{array}{l}\text { WV-03 } \\
0.3 \mathrm{P} \\
1.24 \mathrm{M} \\
\end{array}$ \\
\hline 9. Canary Islands (Spain) & $28.139039,-16.796631$ & Aug-Nov ${ }^{95}$ & Mar 10, 2017 & Migrating & $\begin{array}{l}\text { WV-02 } \\
0.46 \mathrm{P} \\
1.84 \mathrm{M}\end{array}$ \\
\hline 10. Japanese coast (Japan) & $41.947425,143.246413$ & $\mathrm{Apr}^{96}$ & Oct 5,2014 & Migrating & $\begin{array}{l}\text { WV-02 } \\
0.46 \mathrm{P} \\
1.84 \mathrm{M}\end{array}$ \\
\hline
\end{tabular}

Table 2. Sources and characteristics of the images used for the external verification of the whale presence detection model (step-1). Location of the ten evaluated sites. Match between the known whale watching period from the literature and the acquisition date and season of the satellite images in Google Earth. For each image source in Google Earth (reduced spectral resolution) these metadata are provided: the satellite (GE-01: GeoEye-01; QB-02: QuickBird-2; SPOT-6; USGS: United States Geological Survey orthoimagery; WV-02: WorldView-2; and WV-03: WorldView-3), the pixel size at nadir in $\mathrm{m}$, and the sensor spectral resolution (M: Multispectral; P: Panchromatic).

in classification and detection on medium-quality images, such as those available for free in Google Earth. In addition, the CNN-based models are robust ${ }^{59}$ against the differences in spatial and illumination angles across the different satellite sensors used in Google Earth ${ }^{38}$. Automatic image classification methods with convolutional neural networks can save time with respect to manual visual image classification methods ${ }^{60}$. In addition, human fatigue conditions the efficiency of labeling images ${ }^{61}$.

The use of free Google Earth imagery is convenient but it also has limitations since these are RGB images rather than multispectral, only available for few dates that may not be within the known whale presence period, are generally constrained to limited locations along coastal areas (up to $\sim 4 \mathrm{~km}$ offshore), and are restricted for massive access. These last three limitations must be overcome together with the use of supercomputing for the worldwide "wall-to-wall" application of this method but do not impede its use for local assessments of whales around the world. Image spatial resolution can also limit the application of this method to detect cetaceans shorter than $5 \mathrm{~m}$ long (e.g. pilot whales, dolphins, etc.), which would require pixel sizes smaller than $1 \mathrm{~m}$. For example, in our study, higher resolution images tend to give higher F1-measure (Table S3), though low contrast between whales and surrounding water tend to decrease performance (e.g. New Zealand) and high contrast to improve it (Table S3).

Our results showed that the behaviour and the image acquisition date can also bias the probability of detecting whales. The spatial pattern of whales under blowing, breaching, and peduncle postures showed better detectability than under logging and submerged, when whale bodies can be confused with submerged rocks and seafloor. However, the greater number of whales (both detected by the model and photo-interpreted) in our study were under passive (logging and submerged) instead of active behaviour, and in images captured during the breeding season. Therefore, the best time to identify whales might be along the breeding season (Table 1), when whales spend more time in surface and in shallow waters ${ }^{62}$. The effect of overlapped positions between females and calves on their detectability and counting should be further studied. In contrast, the most difficult time might be during migration and in the feeding season (Table 1), when whales are mainly in spyhopping, peduncle, and deeply submerged postures ${ }^{63}$, and in areas with low contrast between water and whales, or under high sea surface roughness, sea glint, or bad atmospheric conditions (clouds or aerosols).

The application of CNNs in remote sensing opens a world of possibilities for biodiversity science and conservation $^{64,65}$. The great performance obtained by the CNN-based models trained on and applied to free VHR images opens the possibility to automatically process millions of satellite images around the world from whale hotspots, marine protected areas, whale sanctuaries, or migratory routes. Our procedure requires less time and lower cost 


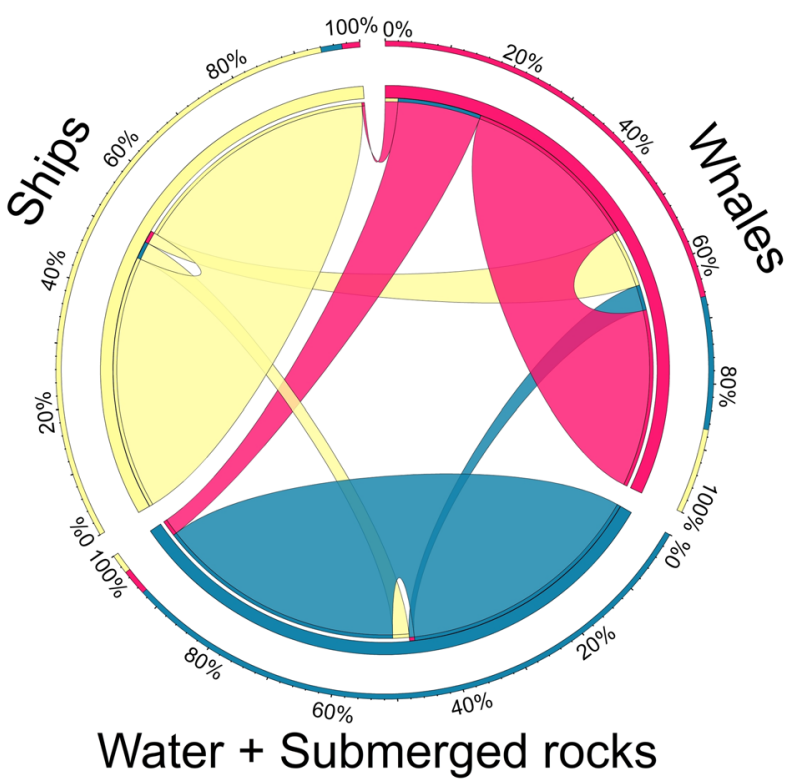

Figure 3. Visualization (Circos plot) of the confusion matrix between the photo-interpreted ground truth and the predictions made by the CNN-based model (step-1) for detecting the presence of whales (in red), ships (yellow), and water + submerged rocks (blue). The links between classes depicts false negatives (whales that were misclassified as ship or water + submerged rock) and false positives (ships or water + submerged rocks that were misclassified as whales), the thickness of these links indicate the percentage of misclassified instances. Errors and successes are shown as a percentage on the outer concentric bars. Only 13 and 1 whale images were classified respectively as water + submerged rocks and as ships, while only 9 ship images and 4 water + submerged rocks images were classified as whales.

than the traditional acoustic surveys from ships or the visual surveys from planes and helicopters. The efficiency of remote sensing methods is particularly relevant to save time and money for long-term whale monitoring in remote places, or under difficult circumstances such as whales trapped inside sea ice in polar regions ${ }^{66}$. The detection of whales using satellite images was already achieved using classical methods ${ }^{29}$, but their portability to other regions or dates was strongly limited by the necessity of spectral normalization. However, our CNN-based model is easily transferable to any region or RGB image with different characteristics in color, lighting and atmospheric conditions, background, or size and shape of the target objects, and it requires no human supervision, which speeds up the detection process ${ }^{37}$.

Further research could increase the performance and variety of species identified by our CNN-model. For instance, the model could be improved by increasing the number of samples and variety of atmospheric and sea conditions in the training datasets, by building hierarchical training datasets with different behaviour across different species ${ }^{67}$, by using more spectral bands and temporal information ${ }^{68}$, and by artificially increasing the spatial resolution of the images through rendering ${ }^{69}$. In addition, as it is a fast and scalable method, it can even be transferred to very high spatial resolution images $(<10 \mathrm{~cm})$ captured by unmanned aerial vehicles (UAVs) for the automatic identification of specific individuals ${ }^{70}$.

A global operationalization of our satellite-based model for whale detection and counting could greatly complement traditional methods ${ }^{12-22}$ to assist whale conservation, to guide marine spatial planning ${ }^{71}$, or to assess regional $^{11}$ and global ${ }^{72}$ priorities for marine biodiversity protection against global change ${ }^{73}$. In addition, our method could be extended to higher resolution RGB images in particular and VHR multispectral data in general to identify and quantify cetaceans species ${ }^{35}$ and other marine species such as seals and sea lions ${ }^{74}$, penguins $s^{75}$, etc. To boost this process, free access to satellite data is $\mathrm{key}^{76}$. The compromise with biodiversity conservation from corporations such as Google, Microsoft, Planet, Airbus, or DigitalGlobe ${ }^{77}$ could be materialized through the systemic release of free high resolution aerial and satellite imagery at least from key sites for marine conservation. Even more, the acquisition of these images in pelagic environments does not directly compete with satellites commercial activity, which is usually focused on terrestrial and coastal areas. Having these images available would also make it possible to organize the development of a global database of images of cetaceans and many other marine vertebrates that could be used to improve the training of our whale detection and counting model or to develop similar models for other marine organisms. Images of the highest spatial resolution (such as WorldView-3 satellite images with a pixel size of $0.3 \mathrm{~m}$ ) are particularly appropriate for this purpose. This way, satellite and CNN-based detectors of big marine organisms could serve to produce global characterizations of species populations and traits and of community composition as part of the initiative by the Group on Earth Observations - Biodiversity Observation Network (GEOBON) on satellite remote sensing essential biodiversity variables $^{78}$. 
A

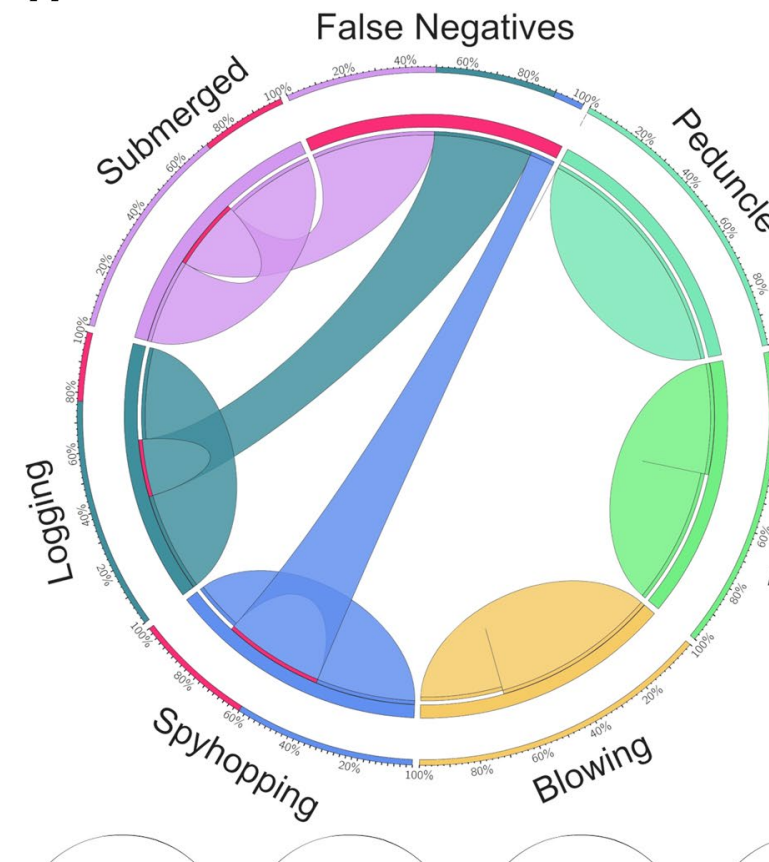

B
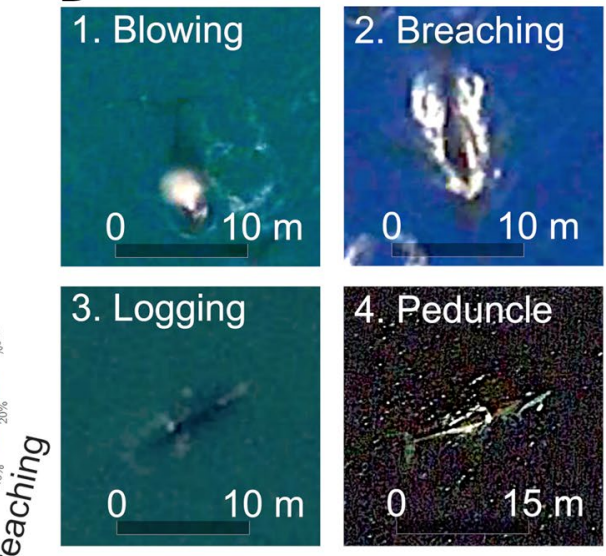

5. Spyhopping
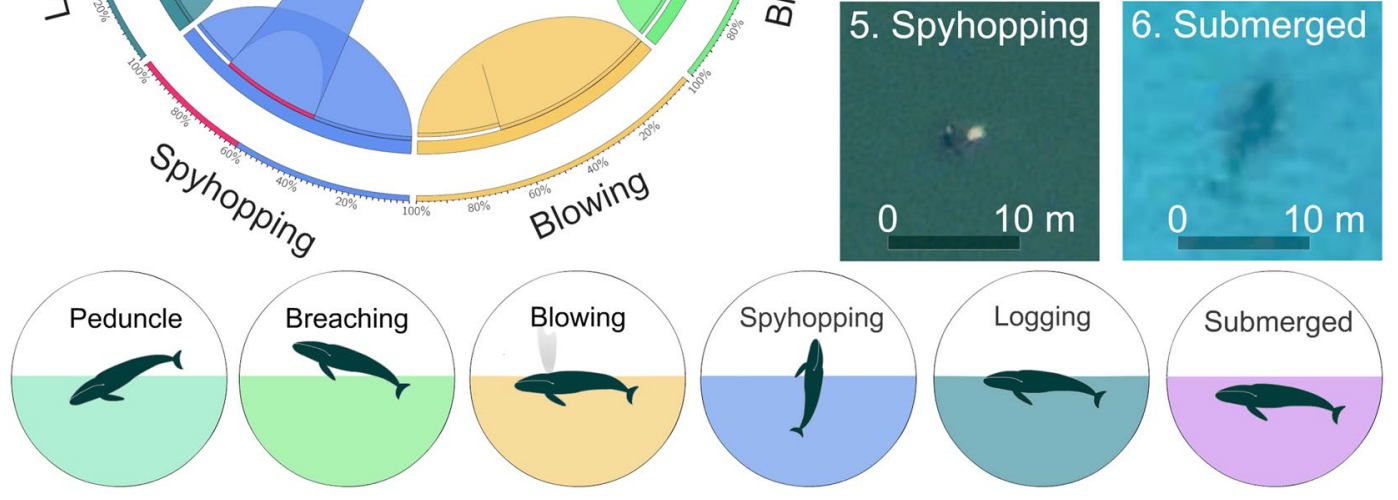

Figure 4. Impact of whale postures or behaviour on the performance of the step-1 CNN-based model for detecting the presence of whales. (A) The Circos plot shows the distribution of the false negatives (undetected whales, in red color) and true positives across whale postures. Whales under blowing, breaching, and peduncle postures were better detected than under spyhopping, logging and submerged. (B) Example of images for each behaviour from the detected hotspots at the highest zoom. Map data: Google, DigitalGlobe.

\section{Methods}

We address the problem of whale counting in large scale areas represented by a large number of VHR satellite and airborne images using a two-step approach that combines two models: (i) an image classification model and (ii) a whale detection model. To build these models, we needed to build two training datasets, one for each model. In this section, we first present the proposed two-step approach for whale counting then describe the process we used for building the training and test datasets for each step. In addition, we compare the performance of our two-step approach with a baseline approach based only on the detection model (Faster RCNN).

The proposed two-step approach. Counting whales in large scale areas that can be represented by a large number of images is not only a complex task but also expensive from a computational point of view. To overcome these limitations, we propose a two-step CNN-based approach capable of counting whales in vast areas with a reduced computational cost, where the first $\mathrm{CNN}$ is used to filter out water potential false positives (ships, foam and rocks) but keeping candidate images to be analyzed later by the second and much slower CNN. To overcome these limitations, we designed a two-step whale counting approach, where the first and quicker CNN filters out images of just water or containing potential false positives (e.g., ships, foam, and rocks) but keeping input images with whale presence, and the second and slower $\mathrm{CNN}$ locates and counts each whale in the latter images. Thanks to this combination of two CNNs, our model is capable of counting whales in vast areas with a reduced computational cost. In our proof of concept, the first step CNN-based model analyzes 10 whale hotspots around the world represented by 13,348 grid-cells using a $71 \times 71 \mathrm{~m}$ sliding window -twice the size of blue whales $(30 \mathrm{~m})$ - and outputs the probability of having detected whales in each cell (Fig. 6A). To reduce the computational cost of the overall approach, the second step CNN-based model analyzes only those cells with high probability of whale presence, localizes each whale within a bounding box, and outputs the number of counted individuals (Fig. 6B). On average, step-1 was less time consuming than step-2 by one order of magnitude (while step-1 only took around 1.02 seconds/image, step- 2 took around 12.35 seconds/image, both in a laptop with a $1.6 \mathrm{GHz}$ i5 CPU and 8 GB of RAM).

To facilitate its use and to support whale conservation, the CNN-based model was built using open-source software and can be used on free Google Earth images (subjected to terms of service). To increase the volume of the training dataset, we used data-augmentation techniques by applying rotation with a factor selected randomly 


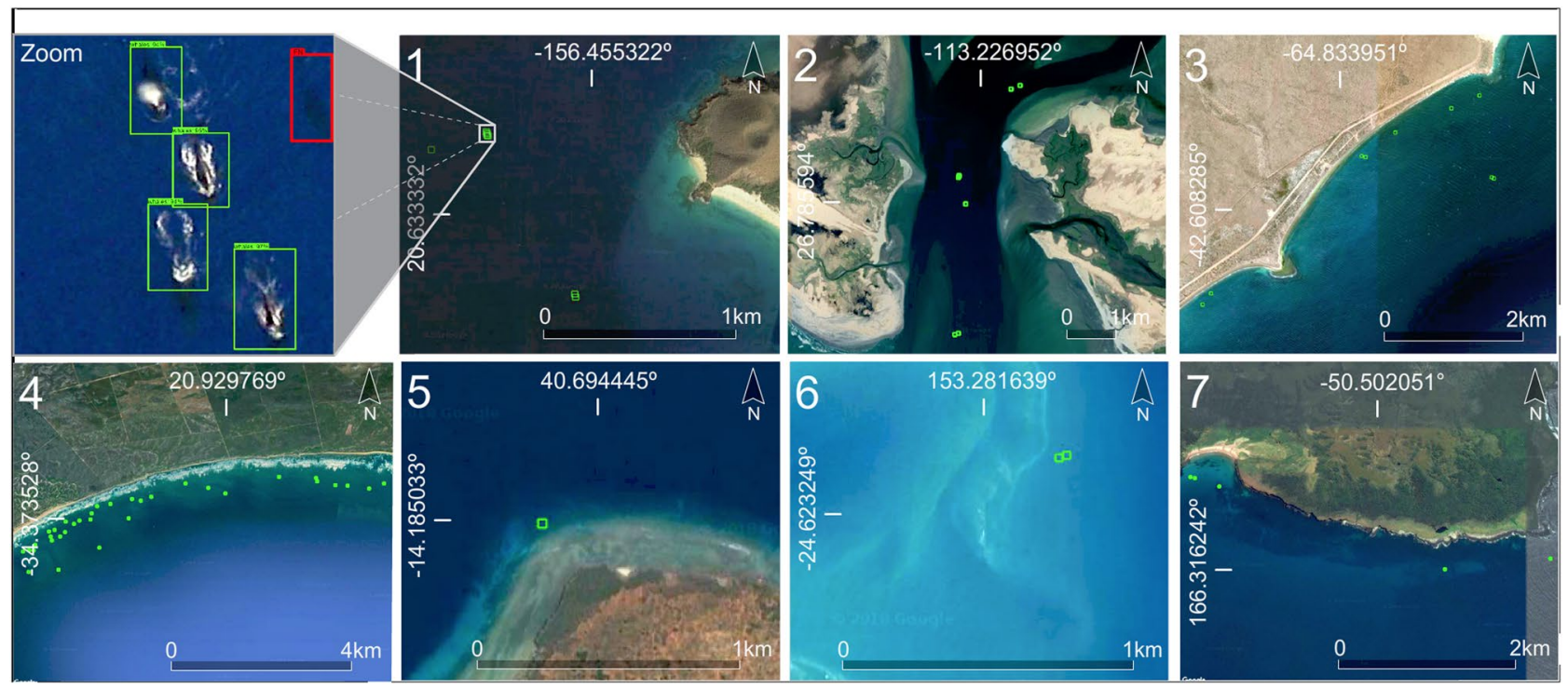

Figure 5. The results of the whale counting (step-2) CNN-based model that locates and counts the number of whales (green bounding boxes) in the grid cells in which step-1 CNN gave high probability for whale presence. The red bounding box shows a false negative. Map data: Google, DigitalGlobe.

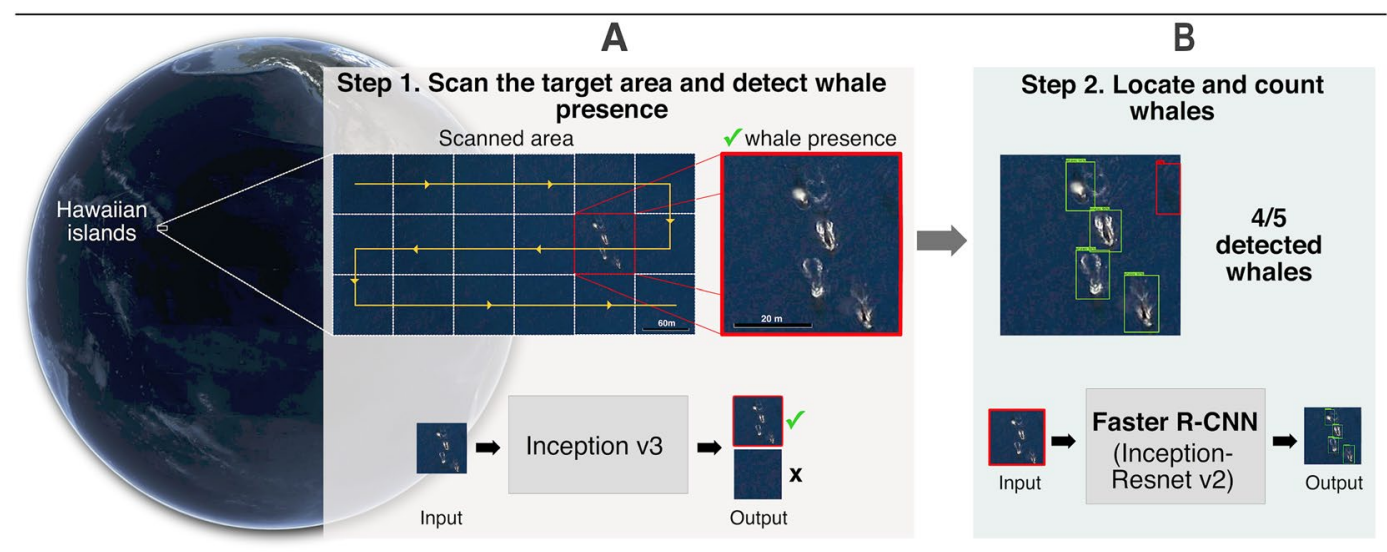

Figure 6. The proposed automatic whale-counting procedure with a two-step CNN-based model. (A) The first-step CNN scans the sample area (following the yellow line) to search for the presence of whales in each grid cell (white squares). Only grid cells in which the first-step CNN gives high probability for whale presence (red square) are analyzed by (B) the second-step $\mathrm{CNN}$, which finally locates and counts individuals (the four green bounding boxes indicate correctly detected whales and the red box indicates a false negative). Map data: Google, DigitalGlobe.

between 0 and $360^{\circ}$, randomly flipping half of the training images, randomly cropping, random the scale size of the images, and random the brightness level of pixels by a factor of up to $50 \%$.

We used Google TensorFlow deep-learning framework ${ }^{79}$ to train, validate and test the step-1 CNN-based model, and Google Tensorflow Object Detection $\mathrm{API}^{80}$ to train, validate and test the step-2 CNN-based model.

Step-1: Whale presence detection phase. When seen from space, whales are often confused with other object classes such as ships and wave foam around partially or entirely submerged rocks. To give the first step $\mathrm{CNN}$-based model the capacity to distinguish between these objects, we addressed the problem as a three-class image classification task. The first model was built using the last version of GoogleNet Inception v3 CNN architecture $^{41}$, pretrained on the massive ImageNet database (around 1.28 million images, organized into 1,000 object categories). We retrained the parameters of the two last fully connected layers in the network on our dataset, using a learning rate of 0.001 and a decay factor of 16 every 30 epochs. As optimization algorithm, we used RMSProp with a momentum and decay of 0.9 and epsilon of 0.1 .

To assess whether whale posture, season, and location affected whale presence detection in satellite images, we compared the F1-measure metric across different seasons and locations of the world, and across multiple active and resting behaviour ${ }^{64}$. 


\begin{tabular}{|l|l|l|}
\hline Accuracy indicator & Equation & Interpretation \\
\hline Positive predictive value (or precision) & $\frac{\text { truepositives }}{\text { truepositives }+ \text { falsepositives }}$ & In how many images the assigned class was correct. \\
\hline Sensitivity (or recall) & $\frac{\text { truepositives }}{\text { truepositives }+ \text { falsenegatives }}$ & From all images of a class, how many were correctly are detected. \\
\hline F1-measure (or F1-score) & $2 x \frac{\text { positivepredictivevaluexsensitivity }}{\text { positivepredictivevalue }+ \text { sensitivity }}$ & Index that evaluates the balance between precision and recall. \\
\hline
\end{tabular}

Table 3. Accuracy indicator, equation, and interpretation of the performance assessment.

Step-2: Whale counting phase. We built the second CNN-based model that counts whales by reformulating the problem into an object detection task. We used the detection model Faster R-CNN based on Inception-Resnet v2 CNN architecture ${ }^{42,81}$, pre-trained on the well known COCO (Common Objects in Context) detection dataset, which contains more than 200,000 images organized into 80 object categories ${ }^{82}$. The two last fully connected layers of the network were retrained on our dataset using a learning rate of 0.001 and a decay factor of 16 every 30 epochs. As optimization algorithm, we used RMSProp with a momentum of 0.9 and epsilon of 0.1 .

Training, testing and validating datasets. Currently, there does not exist any accessible datasets of satellite or aerial RGB images for whales detection. We had to build two datasets for training the CNN-based models to respectively detect the presence of whales and count their number, and a third dataset for testing and validating the whole procedure. We built the training datasets using satellite and aerial images of different resolutions so that the models can generalize correctly to different resolutions, contrasts and colors. The three datasets were built by combining, preprocessing and labeling images selected from the only sources available to us: Google Earth ${ }^{38}$, free Arkive $^{83}$, NOAA Photo Library ${ }^{84}$, and NWPU-RESISC45 dataset ${ }^{85}$. For step-1, the training dataset contains 2,100 images of the following three classes (700 images per class): (1) whales, (2) ships, and (3) "water + submerged rocks" (Data S1). Whale images for training the CNN were mainly aerial images. For step-2, the training dataset contains 700 aerial images, with whales and background, in which each whale is annotated within a bounding box (the total number of bounding boxes is 945).

The dataset for testing and validating the whole procedure consists of RGB (Red, Green, and Blue bands) images downloaded from Google Earth in 14,148 cells of $71 \times 71 \mathrm{~m}$ distributed worldwide. For ships, we selected 400 images from 100 seaports around the world (Data S2). For "water + submerged rocks" class, we selected 400 coastal images randomly around the world (Data S3). Finally, for whales (Table 1), we downloaded 13,348 cells (Data S4) of $71 \mathrm{~m} \times 71 \mathrm{~m}$ from 10 areas that had very high-resolution images at zoom 18 (eye altitude of $\sim 254 \mathrm{~m}$ ) and that are known for marine mammal diversity or whale watching. These areas have been highlighted either as global marine biodiversity hotspots ${ }^{86}$, marine mammal hotspots ${ }^{72}$, irreplaceable or priority conservation sites (threshold $>=0.3)^{11}$, and are included within or next to a marine protected area ${ }^{87}$ (Table 1 ). Two authors visually inspected all the images to annotate each cell with the name of the corresponding class and with the number of whales. From the 13,348 cells in the 10 hotspots for whale watching, the authors' visual photo-interpretation revealed whales only in 68 cells.

The annotators also verified the presence of whales in these areas through specialized websites on whale watching and used the time-lapse tool of Google Earth to differentiate whales from sea floor and submerged rocks by comparing images from the same spot at different dates. Finally, to assess the effect of whale posture or behaviour on model performance, the annotators tagged each of the 68 cells with whale presence with the most dominant or conspicuous posture in it, by choosing from the following active and resting behaviour ${ }^{88}$, i.e., logging, breaching, spyhopping, blowing, peduncle, and submerged.

Comparison between our two-step approach and the baseline detection model (Faster R-CNN). For comparison purposes, we trained and analyzed Faster RCNN directly on the input images without any previous analysis. On the same test images (ten hotspots), Faster RCNN obtains an average F1 42\%, which is substantially lower than the results obtained by our two-step approach (see Table S1D). This low performance is mainly due to the high number of false positives (e.g. boats, foam, rocks), specially in the sites with lower resolution images. Whereas, in site with very high resolution images, i.e., Hawaiian Islands (USA), Faster R-CNN obtained comparable results with our two-step approach, with F1 of $94 \%$. The main reason why our two-step approach reaches much better accuracy than the detection model alone is that step-1 filters out most possible false positives, which consequently helps the next stage, step-2, in finding whales more accurately.

Metrics used in the performance assessment. To evaluate the performance of both CNN-based models, we used these metrics ${ }^{39}$ : positive predictive value, sensitivity, and F1-measure (Table 3).

True positives correspond to images that were correctly classified or counted as whales by the models, false positives correspond to images that were classified or counted as whales by the models but actually corresponded to another class, and false negatives correspond to undetected images with whales. In simple terms, high positive predictive value means that the model returned substantially more actual whales than false ones, while high sensitivity means that the model returned most of the actual whales. F1-measure provides a balance between precision and sensitivity. We used 5-fold Cross-Validation strategy to evaluate our two-step approach and the baseline on the test dataset. 


\section{Data Availability}

The test and training datasets that support our findings are available from Github archive (https://github.com/ EGuirado/CNN-Whales-from-Space). Restrictions apply to the availability of the images of the training and validation data, which were used with permission for the current study, but are not publicly available. Some data may be available from the authors upon reasonable request and under written permission from Google Earth, Arkive, or NOAA Photo Library when applicable. To allow reproducibility, in the Supplementary Information, we provide the metadata of all the images in the training and testing datasets.

\section{References}

1. Payne, R., Rowntree, V., Perkins, J. S., Cooke, J. G. \& Lankester, K. Population size, trends and reproductive parameters of right whales (Eubalaena australis) off Peninsula Valdes, Argentina. Rep. Int. Whal. Comm. 12, 271-278 (1990).

2. Corkeron, P. J. Whale Watching, Iconography, and Marine Conservation. Conserv. Biol. 18, 847-849 (2004).

3. Parsons, E. C. M. et al. Key research questions of global importance for cetacean conservation. Endanger. Species Res. 27, 113-118 (2015).

4. O'Connor, S., Campbell, R., Cortez, H. \& Knowles, T. Whale Watching Worldwide: tourism numbers, expenditures and expanding economic benefits, a special report from the International Fund for Animal Welfare. Yarmouth MA USA Prep. Econ. Large 228 (2009).

5. Bowen, W. D. Role of marine mammals in aquatic ecosystems. Mar. Ecol. Prog. Ser. 158, 267-274 (1997).

6. Smith, L. V. et al. Preliminary investigation into the stimulation of phytoplankton photophysiology and growth by whale faeces. J. Exp. Mar. Biol. Ecol. 446, 1-9 (2013).

7. Roman, J. et al. Whales as marine ecosystem engineers. Front. Ecol. Environ. 12, 377-385 (2014).

8. Jenkins, L. \& Romanzo, C. Makah whaling: aboriginal subsistence or a stepping stone to undermining the commercial whaling moratorium. Colo J Intl Envtl Pol 9, 71 (1998).

9. Jetz, W. \& Freckleton, R. P. Towards a general framework for predicting threat status of data-deficient species from phylogenetic, spatial and environmental information. Philos. Trans. R. Soc. B Biol. Sci. 370, 20140016 (2015).

10. Braulik, G. T., Findlay, K., Cerchio, S., Baldwin, R. \& Perrin, W. Sousa plumbea. The IUCN Red List of Threatened Species 2017: e. T82031633A82031644. (2017).

11. Jenkins, C. N. \& Van Houtan, K. S. Global and regional priorities for marine biodiversity protection. Biol. Conserv. 204, 333-339 (2016).

12. Perrin, W. F., Würsig, B. \& Thewissen, J. G. M. Encyclopedia of marine mammals. (Academic Press, 2018).

13. Harcourt, R., van der Hoop, J., Kraus, S. \& Carroll, E. L. Future directions in Eubalaena spp.: comparative research to inform conservation. Front. Mar. Sci. (2019).

14. Barlow, J. \& Gerrodette, T. Abundance of cetaceans in California waters based on 1991 and 1993 ship surveys. US Dep. Commer. NOAA Tech. Memo. NOAA-TM-NMFS-SWFSC-233 (1996).

15. Hammond, P. S. et al. Estimates of cetacean abundance in European Atlantic waters in summer 2016 from the SCANS-III aerial and shipboard surveys. (Wageningen Marine Research, 2017).

16. Pike, D. G., Paxton, C. G., Gunnlaugsson, T. \& Víkingsson, G. A. Trends in the distribution and abundance of cetaceans from aerial surveys in Icelandic coastal waters, 1986-2001. NAMMCO Sci. Publ. 7, 117-142 (2009).

17. Kingsley, M. C. S. \& Reeves, R. R. Aerial surveys of cetaceans in the Gulf of St. Lawrence in 1995 and 1996. Can. J. Zool. 76, $1529-1550$ (1998).

18. Evans, P. G. \& Hammond, P. S. Monitoring cetaceans in European waters. Mammal Rev. 34, 131-156 (2004).

19. Panigada, S., Lauriano, G., Burt, L., Pierantonio, N. \& Donovan, G. Monitoring winter and summer abundance of cetaceans in the Pelagos Sanctuary (northwestern Mediterranean Sea) through aerial surveys. PloS One 6, e22878 (2011).

20. Charry, B., Marcoux, M. \& Humphries, M. M. Aerial photographic identification of narwhal (Monodon monoceros) newborns and their spatial proximity to the nearest adult female. Arct. Sci. 4, 513-524 (2018).

21. Mellinger, D. K., Stafford, K. M., Moore, S. E., Dziak, R. P. \& Matsumoto, H. An overview of fixed passive acoustic observation methods for cetaceans. Oceanography 20, 36-45 (2007).

22. Marques, T. A., Munger, L., Thomas, L., Wiggins, S. \& Hildebrand, J. A. Estimating North Pacific right whale Eubalaena japonica density using passive acoustic cue counting. Endanger. Species Res. 13, 163-172 (2011).

23. Barlow, J. \& Taylor, B. L. Estimates of sperm whale abundance in the northeastern temperate Pacific from a combined acoustic and visual survey. Mar. Mammal Sci. 21, 429-445 (2005).

24. Fleming, A. H. et al. Combining acoustic and visual detections in habitat models of Dall's porpoise. Ecol. Model. 384, 198-208 (2018).

25. Kennedy, A. S. et al. Local and migratory movements of humpback whales (Megaptera novaeangliae) satellite-tracked in the North Atlantic Ocean. Can. J. Zool. 92, 9-18 (2013).

26. Wade, P. et al. Acoustic detection and satellite-tracking leads to discovery of rare concentration of endangered North Pacific right whales. Biol. Lett. 2, 417-419 (2006).

27. Schweder, T., Sadykova, D., Rugh, D. \& Koski, W. Population estimates from aerial photographic surveys of naturally and variably marked bowhead whales. J. Agric. Biol. Environ. Stat. 15, 1-19 (2010).

28. Hodgson, A., Kelly, N. \& Peel, D. Unmanned aerial vehicles (UAVs) for surveying marine fauna: a dugong case study. PloS One 8, e79556 (2013).

29. Abileah, R. Marine mammal census using space satellite imagery. US Navy J. Underw. Acoust. 52 (2002).

30. Aniceto, A. S. et al. Monitoring marine mammals using unmanned aerial vehicles: quantifying detection certainty. Ecosphere 9, e02122 (2018).

31. Taylor, B. L., Martinez, M., Gerrodette, T., Barlow, J. \& Hrovat, Y. N. Lessons from Monitoring Trends in Abundance of Marine Mammals. Mar. Mammal Sci. 23, 157-175 (2007).

32. Kerr, J. T. \& Ostrovsky, M. From space to species: ecological applications for remote sensing. Trends Ecol. Evol. 18, 299-305 (2003).

33. Fretwell, P. T., Staniland, I. J. \& Forcada, J. Whales from Space: Counting Southern Right Whales by Satellite. PLOS ONE 9 , e88655 (2014).

34. Rose, R. A. et al. Ten ways remote sensing can contribute to conservation. Conserv. Biol. 29, 350-359 (2015).

35. Cubaynes, H. C., Fretwell, P. T., Bamford, C., Gerrish, L. \& Jackson, J. A. Whales from space: Four mysticete species described using new VHR satellite imagery. Mar. Mammal Sci. 35, 466-491 (2019).

36. Ren, S., He, K., Girshick, R. \& Sun, J. Faster R-CNN: Towards Real-Time Object Detection with Region Proposal Networks. in Advances in Neural Information Processing Systems 28 (eds Cortes, C., Lawrence, N. D., Lee, D. D., Sugiyama, M. \& Garnett, R.) 91-99 (Curran Associates, Inc., 2015).

37. LeCun, Y., Bengio, Y. \& Hinton, G. Deep learning. Nature 521, 436-444 (2015).

38. Yu, L. \& Gong, P. Google Earth as a virtual globe tool for Earth science applications at the global scale: progress and perspectives. Int. J. Remote Sens. 33, 3966-3986 (2012). 
39. Guirado, E., Tabik, S., Alcaraz-Segura, D., Cabello, J. \& Herrera, F. Deep-learning Versus OBIA for Scattered Shrub Detection with Google Earth Imagery: Ziziphus lotus as Case Study. Remote Sens. 9, 1220 (2017).

40. Lecun, Y., Bottou, L., Bengio, Y. \& Haffner, P. Gradient-based learning applied to document recognition. Proc. IEEE 86, 2278-2324 (1998).

41. Szegedy, C., Vanhoucke, V., Ioffe, S., Shlens, J. \& Wojna, Z. Rethinking the Inception Architecture for Computer Vision. In Proc. CVPR IEEE 2818-2826 (2016).

42. Szegedy, C., Ioffe, S., Vanhoucke, V. \& Alemi, A. Inception-v4, Inception-ResNet and the Impact of Residual Connections on Learning. ArXiv160207261 Cs (2016).

43. Redmon, J. \& Farhadi, A. YOLO9000: Better, Faster, Stronger. In Proc. CVPR IEEE 7263-7271 (2017).

44. Lin, T.-Y. et al. Feature Pyramid Networks for Object Detection. in Proc. CVPR IEEE 2117-2125 (2017).

45. Zhang, S., Wen, L., Bian, X., Lei, Z. \& Li, S. Z. Single-Shot Refinement Neural Network for Object Detection. in Proc. CVPR IEEE 4203-4212 (2018).

46. Fu, C.-Y., Liu, W., Ranga, A., Tyagi, A. \& Berg, A. C. DSSD: Deconvolutional Single Shot Detector. ArXiv170106659 Cs (2017).

47. Lin, T.-Y., Goyal, P., Girshick, R., He, K. \& Dollár, P. Focal Loss for Dense Object Detection. ArXiv170802002 Cs (2017).

48. Zhang, X. et al. Geospatial Object Detection on High Resolution Remote Sensing Imagery Based on Double Multi-Scale Feature Pyramid Network. Remote Sens. 11, 755 (2019).

49. Guo, W., Yang, W., Zhang, H. \& Hua, G. Geospatial Object Detection in High Resolution Satellite Images Based on Multi-Scale Convolutional Neural Network. Remote Sens. 10, 131 (2018).

50. Ji, H., Gao, Z., Mei, T. \& Li, Y. Improved Faster R-CNN With Multiscale Feature Fusion and Homography Augmentation for Vehicle Detection in Remote Sensing Images. IEEE Geosci. Remote Sens. Lett. 1-5, https://doi.org/10.1109/LGRS.2019.2909541 (2019).

51. Huang, J. et al. Speed/Accuracy Trade-Offs for Modern Convolutional Object Detectors. in Proc. CVPR IEEE 7310-7311 (2017).

52. Shelden, K. E. W. \& Laake, J. L. Comparison of the offshore distribution of southbound migrating gray whales from aerial survey data collected off Granite Canyon, California, 1979-96. J Cetacean Res Manag 5, 53-56 (2002).

53. Rowat, D., Gore, M., Meekan, M. G., Lawler, I. R. \& Bradshaw, C. J. A. Aerial survey as a tool to estimate whale shark abundance trends. J. Exp. Mar. Biol. Ecol. 368, 1-8 (2009).

54. Esteva, A. et al. Dermatologist-level classification of skin cancer with deep neural networks. Nature 542, 115-118 (2017).

55. Silver, D. et al. Mastering the game of Go with deep neural networks and tree search. Nature 529, 484-489 (2016).

56. Mnih, V. et al. Human-level control through deep reinforcement learning. Nature 518, 529-533 (2015).

57. Dodge, S. \& Karam, L. Understanding how image quality affects deep neural networks. In 2016 Eighth International Conference on Quality of Multimedia Experience (QoMEX) 1-6, https://doi.org/10.1109/QoMEX.2016.7498955 (2016).

58. Kim, J., Lee, J. K. \& Lee, K. M. Accurate Image Super-Resolution Using Very Deep Convolutional Networks. in Proc. CVPR IEEE 1646-1654, https://doi.org/10.1109/CVPR.2016.182 (2016).

59. Tabik, S., Peralta, D., Herrera-Poyatos, A. \& Herrera, F. A snapshot of image pre-processing for convolutional neural networks: case study of MNIST. Int. J. Comput. Intell. Syst. 10, 555-568 (2017).

60. Norouzzadeh, M. S. et al. Automatically identifying, counting, and describing wild animals in camera-trap images with deep learning. Proc. Natl. Acad. Sci. 115, E5716-E5725 (2018).

61. Maksimenko, V. A. et al. Increasing Human Performance by Sharing Cognitive Load Using Brain-to-Brain Interface. Front. Neurosci. $12(2018)$

62. Norris, K. S. \& Sciences, A. I. of B. The seasonal migratory cycle of humpback whales. In Whales, Dolphins, and Porpoises 145-171 (University of California Press, 1966).

63. Corkeron, P. J. Humpback whales (Megaptera novaeangliae) in Hervey Bay, Queensland: behaviour and responses to whalewatching vessels. Can. J. Zool. 73, 1290-1299 (1995).

64. Lyamin, O. I., Manger, P. R., Mukhametov, L. M., Siegel, J. M. \& Shpak, O. V. Rest and activity states in a gray whale. J. Sleep Res. 9, 261-267 (2000).

65. Su, J.-H., Piao, Y.-C., Luo, Z. \& Yan, B.-P. Modeling Habitat Suitability of Migratory Birds from Remote Sensing Images Using Convolutional Neural Networks. Anim. Open Access J. MDPI 8 (2018).

66. Williams, R. et al. Counting whales in a challenging, changing environment. Sci. Rep. 4, 4170 (2014)

67. Yan, Z. et al. HD-CNN: Hierarchical Deep Convolutional Neural Networks for Large Scale Visual Recognition. in 2015 IEEE International Conference on Computer Vision (ICCV) 2740-2748, https://doi.org/10.1109/ICCV.2015.314 (2015).

68. Basaeed, E., Bhaskar, H. \& Al-Mualla, M. CNN-based multi-band fused boundary detection for remotely sensed images. In 6 th International Conference on Imaging for Crime Prevention and Detection (ICDP-15) 1-6, https://doi.org/10.1049/ic.2015.0109 (2015).

69. Fang, L., Au, O. C., Tang, K. \& Wen, X. Increasing image resolution on portable displays by subpixel rendering - a systematic overview. APSIPA Trans. Signal Inf. Process. 1 (2012).

70. Apprill, A. et al. Extensive Core Microbiome in Drone-Captured Whale Blow Supports a Framework for Health Monitoring. mSystems 2, e00119-17 (2017).

71. Augé, A. A. et al. Framework for mapping key areas for marine megafauna to inform Marine Spatial Planning: The Falkland Islands case study. Mar. Policy 92, 61-72 (2018).

72. Pompa, S., Ehrlich, P. R. \& Ceballos, G. Global distribution and conservation of marine mammals. Proc. Natl. Acad. Sci. 108, 13600-13605 (2011).

73. Hays, G. C. et al. Key Questions in Marine Megafauna Movement Ecology. Trends Ecol. Evol. 31, 463-475 (2016)

74. Moxley, J. H. et al. Google Haul Out: Earth Observation Imagery and Digital Aerial Surveys in Coastal Wildlife Management and Abundance Estimation. BioScience 67, 760-768 (2017)

75. Lynch, H. J., White, R., Black, A. D. \& Naveen, R. Detection, differentiation, and abundance estimation of penguin species by highresolution satellite imagery. Polar Biol. 35, 963-968 (2012).

76. Buchanan, G. M. et al. Free satellite data key to conservation. Science 361, 139-140 (2018)

77. Popkin, G. Technology and satellite companies open up a world of data. Nature 557, 745 (2018).

78. Pettorelli, N., Owen, H. J. F. \& Duncan, C. How do we want Satellite Remote Sensing to support biodiversity conservation globally? Methods Ecol. Evol. 7, 656-665 (2016)

79. Abadi, M. et al. TensorFlow: Large-Scale Machine Learning on Heterogeneous Distributed Systems. ArXiv160304467 Cs (2016)

80. Huang, J. et al. Speed/accuracy trade-offs for modern convolutional object detectors. ArXiv161110012 Cs (2016).

81. Krizhevsky, A., Sutskever, I. \& Hinton, G. E. ImageNet Classification with Deep Convolutional Neural Networks. In Advances in Neural Information Processing Systems 25 (eds Pereira, F., Burges, C. J. C., Bottou, L. \& Weinberger, K. Q.) 1097-1105 (Curran Associates, Inc., 2012).

82. Lin, T.-Y. et al. Microsoft COCO: Common Objects in Context. In Computer Vision - ECCV 2014 (eds. Fleet, D., Pajdla, T., Schiele, B. \& Tuytelaars, T.) 740-755 (Springer International Publishing, 2014).

83. Arkive, W. Discover the world's most endangered species. Wildscreen Arkive (2018). Available at, http://www.arkive.org. (Accessed: 20th January 2018)

84. NOAA. NOAA, Photo Library. (2018). Available at, http://www.photolib.noaa.gov. (Accessed: 20th January 2018).

85. Cheng, G., Han, J. \& Lu, X. Remote Sensing Image Scene Classification: Benchmark and State of the Art. Proc. IEEE 105, 1865-1883 (2017). 
86. Ramírez, F., Afán, I., Davis, L. S. \& Chiaradia, A. Climate impacts on global hot spots of marine biodiversity. Sci. Adv. 3, e1601198 (2017).

87. UNEP-WCMC. World Database on Protected Areas (WDPA). IUCN (2016). Available at, https://www.iucn.org/theme/protectedareas/our-work/quality-and-effectiveness/world-database-protected-areas-wdpa. (Accessed: 15th July 2018).

88. Kavanagh, A. S. et al. Evidence for the functions of surface-active behaviors in humpback whales (Megaptera novaeangliae). Mar. Mammal Sci. 33, 313-334 (2017).

89. Handbook, W. W. Bias and variability in distance estimation on the water: implications for the management of whale watching. In IWC Meeting Document SC/52/WW1 (2000).

90. Seger, K. D., Thode, A. M., Swartz, S. L. \& Urbán, R. J. The ambient acoustic environment in Laguna San Ignacio, Baja California Sur, Mexico. J. Acoust. Soc. Am. 138, 3397-3410 (2015).

91. Banks, A. Recent sightings of southern right whales in Mozambique. In Paper SC/S11/RW17 presented to the IWC Southern Right Whale Assessment Workshop 21 (2011).

92. Stamation, K. A., Croft, D. B., Shaughnessy, P. D., Waples, K. A. \& Briggs, S. V. Behavioral responses of humpback whales (Megaptera novaeangliae) to whale-watching vessels on the southeastern coast of Australia. Mar. Mammal Sci. 26, 98-122 (2010).

93. Stewart, R. \& Todd, B. A note on observations of southern right whales at Campbell Island, New Zealand. J. Cetacean Res. Manage. 2, 117-120 (2001).

94. Félix, F. \& Botero-Acosta, N. Distribution and behaviour of humpback whale mother-calf pairs during the breeding season off Ecuador. Mar. Ecol. Prog. Ser. 426, 277-287 (2011).

95. Carrillo, M., Pérez-Vallazza, C. \& Álvarez-Vázquez, R. Cetacean diversity and distribution off Tenerife (Canary Islands). Mar. Biodivers. Rec. 3 (2010).

96. Darling, J. D. \& Cerchio, S. Movement of a Humpback Whale (megaptera Novaeangliae) Between Japan and Hawaii. Mar. Mammal Sci. 9, 84-88 (1993).

\section{Acknowledgements}

S.T. was supported by the Ramón y Cajal Programme of the Spanish government (RYC-2015-18136). S.T., E.G., and F.H. were supported by the Spanish Ministry of Science under the project TIN2017-89517-P. D. A-S. received support from European LIFE Project ADAPTAMED LIFE14 CCA/ES/000612, and from ERDF and Andalusian Government under the project GLOCHARID. D.A.-S. received support from NASA Work Programme on Group on Earth Observations - Biodiversity Observation Network (GEOBON) under grant 80NSSC18K0446, from project ECOPOTENTIAL, funded by European Union Horizon 2020 Research and Innovation Programme under grant agreement No. 641762, and from the Spanish Ministry of Science under project CGL2014-61610-EXP and grant JC2015-00316. M.R. received support from International mobility grant for prestigious researchers by (CEIMAR) International Campus of Excellence of the Sea.

\section{Author Contributions}

E.G. and S.T. designed the experiments, collected the data, implemented the code, ran the models, analyzed the results, and wrote the paper. D.A.-S. and M.L.R., provided guidance, contributed to the design of the analyses, analyzed the results, and wrote the paper. F.H. provided guidance, contributed to the design of the study, and wrote the paper.

\section{Additional Information}

Supplementary information accompanies this paper at https://doi.org/10.1038/s41598-019-50795-9.

Competing Interests: The authors declare no competing interests.

Publisher's note Springer Nature remains neutral with regard to jurisdictional claims in published maps and institutional affiliations.

Open Access This article is licensed under a Creative Commons Attribution 4.0 International License, which permits use, sharing, adaptation, distribution and reproduction in any medium or format, as long as you give appropriate credit to the original author(s) and the source, provide a link to the Creative Commons license, and indicate if changes were made. The images or other third party material in this article are included in the article's Creative Commons license, unless indicated otherwise in a credit line to the material. If material is not included in the article's Creative Commons license and your intended use is not permitted by statutory regulation or exceeds the permitted use, you will need to obtain permission directly from the copyright holder. To view a copy of this license, visit http://creativecommons.org/licenses/by/4.0/.

(c) The Author(s) 2019 